\title{
Update on Nonsurgical, Ultraconservative Approaches to Treat Effectively Non-Cavitated Caries Lesions in Permanent Teeth
}

\author{
Boniek Castillo Dutra Borges ${ }^{a}$ \\ Juliane de Souza Borges ${ }^{b}$ \\ Larissa Sgarbosa Napoleao de Araujoc \\ Claudia Tavares Machado ${ }^{\mathrm{a}, \mathrm{d}}$ \\ Alex Jose Souza dos Santos ${ }^{\mathrm{b}, \mathrm{d}}$ \\ Isauremi Vieira de Assunçao Pinheiro ${ }^{b}$
}

\section{ABSTRACT}

Dental caries on tooth surfaces is still a problem in many industrialized countries. For many years, dentistry was influenced by a mechanical approach characterized by the use of high-speed rotary cutting instruments, and dentists predominantly used surgical methods to address caries. This included radical removal of diseased portions of the tooth, along with material-driven geometric extensions to areas that were assumed to be caries-resistant. This concept of extension for prevention was introduced by G. V. Black and influenced dentists for more than 120 years. Recently, a new paradigm of operative conservatism, sometimes referred to as "minimally invasive dentistry," has gained popularity. This paradigm is designed to promote maximum preservation of healthy dental structures over a lifetime. The aim of this review is to discuss the efficacy of current nonsurgical treatments for non-cavitated caries lesions in permanent teeth. Based on results obtained from clinical trials, this review evaluates treatments such as consumption of CPP-ACP added gums, resin infiltration and fissure sealing. Although in a few cases an invasive approach is needed to arrest caries progression, the non-surgical approach generally provides potential benefits that include conserving structure by delaying intervention or minimizing the operative procedure. All current non-invasive methods are effective in treating non-cavitated caries lesions. The adoption of non-invasive approaches in the management of these lesions can preserve dental tissues, thus increasing tooth longevity. (Eur J Dent 2011;5:229-236)

Key words: Dental history; Minimally invasive dentistry; Operative dentistry; Pit-and fissure sealants; Remineralization; Resin infiltration.

a Foundation for Research Support of Rio Grande do Norte (FAPERN), Brazil.

b Department of Dentistry, Federal University of Rio Grande do Norte (UFRN), Brazil.

Department of Restorative Dentistry, Piracicaba Dental School, State University of Campinas (UNICAMP), Brazil. d School of Dentistry, Potiguar University (Laureate International Universities), Brazil.

Corresponding author: Boniek Castillo Dutra Borges Street Minas Novas, 390, cs 18, Natal-RN, Brazil Zip-code: 59088-725

Phone: +55 8432072981 Fax: +55 8432144111

E-mail: boniek.castillodgmail.com 


\section{INTRODUCTION}

It is important to note that the dental profession's approach to the treatment of caries has been evolving in recent years. A generation ago, it would have been fair to characterize the predominant treatment philosophy as being reactive and focusing on operative intervention. When lesions were detected, and often when they were just suspected, they were restored, and the earlier the better. Over the past several decades, advances in materials and technology and changes in caries epidemiology have all contributed to the emergence of a more proactive, tailored, preventive and conservative treatment philosophy characterized by greater attention to the individual and his or her disease, with reduced emphasis on universal immediate surgical intervention. ${ }^{1}$

In a changing practice environment, it is important that educational institutions and providers of continuing education continually update the body of evidence related to the effectiveness of sealants in dental caries prevention and management. ${ }^{2}$ Moreover, other non-surgical therapeutic modalities to treat caries lesions have been raised in the literature, such as resin infiltration ${ }^{3}$ and consumption of CPP-ACP-based products. ${ }^{4} \mathrm{How}-$ ever, fewer studies have discussed the efficacy of these treatments for caries lesions and their benefits on oral health based on results of longterm clinical trials. Therefore, this study aimed to discuss the role of the recent non-surgical, ultraconservative approaches in treating non-cavitated caries lesions by means of clinical investigations in permanent teeth.

\section{DENTAL CARIES: BIOLOGY AND EPIDEMIOLOGY}

Dental caries is a complex, multifactorial, biofilm dependent, and chronic disease process ${ }^{5}$ caused when the products of bacteria diffuse into tooth enamel and dentin and dissolve the mineral. ${ }^{6}$ Caries occurs as a result of a continuum of cyclic demineralization and remineralization of enamel.? Some components of the caries process act at the tooth surface (saliva, biofilm, diet, fluoride), while another set of determinants of the process act at an individual level la person's behavior, knowledge, attitude, education, socioeconomic status, income).$^{8}$ The formation of plaque biofilm enables the proliferation of acidogenic, aciduric, and cariogenic bacteria ${ }^{5}$ and consequently reduces salivary $\mathrm{pH}{ }^{9}$ Frequent consumption of dietary sugars and acidic drinks ${ }^{10}$ throughout the day further prolongs periods of low $\mathrm{pH}^{5}$ Such extended periods cause an overgrowth of pathogenic oral bacteria, which produces acid. Depending on the individual factor above described, the process that act at the tooth surface can lead to a loss of mineral content in the tooth and caries progression into the tooth. 5,8,9

While the prevalence of dental caries worldwide has shown a dramatic decline in recent decades that correlates with the advent of fluoridated water, numerous tooth cleaning devices and products, antibiotic treatment, and professional intervention, dental caries remains the number one chronic oral disease. ${ }^{11,12}$ Occlusal and approximal surfaces of teeth are the most frequent sites found with caries lesions. The biofilm tends to form and mature in certain locations on the tooth, notably the occlusal surface, especially during eruption; the approximal surface cervical to the contact point; and along the gingival margin. These areas are relatively protected from mechanical wear by tongue, cheeks, abrasive food, and toothbrushing. Thus, these are the sites where caries lesions may become visible. It should be noted, however, that there is nothing special chemically about these particular areas of the tooth surface; they are susceptible to lesion development only because the biofilm tends to stagnate there and remain "undisturbed" for prolonged periods of time. ${ }^{13}$ In this sense, clinicians should be able to treat caries lesions in the best way, especially occlusal and approximal ones.

\section{FROM BLACK'S ERA TO THE MINIMALLY INVASIVE DENTISTRY APPROACH}

It has been over 100 years since G. V. Black gathered together most of the knowledge then current on the caries process and set clear parameters for the discipline of operative dentistry. Caries was regarded as "gangrene" of the tooth structure and it was not clear as to whether it began on the outside or the inside of the tooth crown. The roles of bacteria and food stuffs were hotly debated and the materials for restoration of lesions were relatively primitive. $^{14}$

In a farsighted statement, Black postulated that "the causes of immunity and susceptibility to dental 
caries would necessarily be found in conditions of the general system influencing the qualities of the mixed fluids of the mouth by which the teeth are surrounded". ${ }^{15}$ In other words, it was recognized that caries fulfils the dictionary definition of a "disease"; that is "a condition that results in medically significant symptoms in a human." A limited treatment dictated by the lack of depth in the understanding of the etiology was offered. There was an inadequate approach wherein the lesion was simply debrided and restored, without addressing the disease at a more fundamental level. As the disease would probably remain present and active, the cycle of debridement and restoration was likely to be repeated, tooth extraction was the most reliable method of treatment for caries lesions. ${ }^{14}$

By the second half of the twentieth century, the knowledge and understanding of preventive measures had reached a reasonably sophisticated level and the profession was prepared to devote time to patient education. ${ }^{14}$ Research in cariology showed it was essential that the profession understand how the disease is initiated in the oral environment and how it progresses. As early as 1967, attention was drawn to the changing concepts in the treatment of caries. ${ }^{13,16-18}$ After Fusayama and colleagues summarized their concepts on major modifications to cavity designs and the use of a total etch technique using resin composite as the restorative material, it became apparent that the caries process could be arrested and in fact reversed. ${ }^{19}$ It was shown that the powers of dental pulp recovery were greater than had previously been thought. ${ }^{20}$ Science then showed that the fluoride ion is an essential component in all age groups for the control of the disease. In recent times, a technique has been developed that will ensure remineralization of enamel in depth to the extent that it can eliminate the white spot lesion completely. ${ }^{21}$ Moreover, it was demonstrated that glass ionomers are bioactive and will assist in remineralizing dentine on the floor of a caries lesion. ${ }^{22}$

The above-mentioned works contributed to develop the new minimal intervention approach in dentistry. Investigations into the cause and extent of the disease in the individual patient's oral environment are essential corollaries to the treatment, so it is essential to begin with elimination of the disease. ${ }^{14}$ If surgical interference is required, it should be recognized that only that part of the tooth surface which is irreversibly broken down will need to be replaced. Surrounding and underlying demineralized tooth structure can still be remineralized and healed and therefore need not be removed. This means that the G. V. Black mantra of "extension for prevention" should be replaced with the concept of "prevention of extension". ${ }^{14}$ So, minimally invasive operative dentistry is a conservative philosophy based on sound science that spans the breadth of the disease continuum, including nonsurgical management of early non-cavitated carious lesions ${ }^{23}$ and the use of effective conservative interventions for dentinal caries. ${ }^{24}$ In this sense, it also has been recognized that the appropriate management of dental caries should involve improved diagnosis of early noncavitated lesions and treatment for prevention and arrest of such lesions. ${ }^{25}$

\section{TRADITIONAL CARIES}

\section{REMINERALIZING STRATEGIES}

Primary caries lesions are classified as either enamel or dentin lesions. Enamel lesions may be subdivided into outer- and inner-enamel lesions. Caries that have reached dentin are classified as being in either the outer or the inner dentin; alternatively, the dentin lesions may be divided into three depth categories: outer, middle or inner dentin. ${ }^{26}$

Ideally, remineralizing agents need to rapidly precipitate on partially demineralized tooth structure and transform into a more stable, less acidsoluble apatite than the hard tissue replaced. They would need to do this in the presence of saliva and before the next acid challenge comes in contact with the newly precipitated mineral. If the mineral phase that is formed is soluble in saliva or under acid conditions, it will be rapidly lost. On the other hand, mineral that is taken up by the enamel may serve as a reservoir that could be released into fluid phase surrounding the enamel crystals during a caries attack and serve as a substrate for subsequent remineralization. ${ }^{25}$

Delivery methods for remineralization materials include toothpastes, mouth rinses, gels, pastes, chewing gums, lozenges, and foods and beverages. Fluoride remains the best established remineralization strategy, although definite evidence that it can clinically reverse early caries 
lesion remains limited. ${ }^{25}$ Moreover, other techniques of remineralizing or even arresting progression of early enamel and dentin or root caries have been developed.

\section{NOVEL EFFECTIVE TREATMENTS FOR EARLY ENAMEL CARIES}

\section{Remineralizing agents}

The main remineralizer agents under consideration are casein phosphopeptide-amorphous calcium phosphate (CPP-ACP) and casein phosphopeptide-amorphous calcium fluoride phosphate (CPP-ACFP), with major attention for the CPP-ACP. CPP-ACP is a nanocomplex thought to provide a reservoir of calcium and phosphate ions to maintain a state of super-saturation with respect to tooth enamel and buffer plaque $\mathrm{pH}$, and to provide ions for tooth enamel remineralization. ${ }^{25}$ CPP-ACP can be incorporated into products such as mouth washes, chewing gums or professionaluse tooth creams. It has been related that using mouth rinse or chewing sugar-free gums containing CPP-ACP increases the level of calcium phosphate in plaque. ${ }^{27}$

Some authors have investigated the potential benefits of the consumption of gum CPP-ACP added on arresting progression of early dental caries in enamel. It was demonstrated that the use of sugar-free, CPP-ACP-based gum was able to remineralize enamel subsurface lesions in situ. ${ }^{28}$ In this study, specimens containing enamel subsurface lesions were sectioned into test and control half-slabs with the test half-slabs inserted into removable palatal appliances. First, subjects $(n=10)$ chewed one of the three randomly allocated gums ltwo CPP-ACP-free and one CPP-ACP-containingl for a 20-min period four times per day for 14 days. At the end of the study period, each subject had chewed all three test gums, with a 7-day washout period between crossovers. After each 14-day cycle, test and control half-slabs were paired, embedded in resin, sectioned, and subjected to microradiography to determine remineralization. The gum that contained CPP-ACP showed significantly greater remineralization than did the other two gums.

In a 24-month clinical trial, ${ }^{4}$ progression and regression of enamel approximal caries were investigated after 2,720 subjects randomly chewed gums with or without $54 \mathrm{mg}$ of CPP-ACP. The results showed that for subjects chewing the CPP-ACP gum, the odds of a surface experiencing caries progression were $18 \%$ less than those of a surface experiencing caries progression for subjects chewing the control gum. The $54 \mathrm{mg}$ CPP-ACP sugar-free gum significantly slowed progression and enhanced regression of approximal caries relative to a control sugar-free gum in a 24-month clinical trial. Moreover, it was found in situ that CPP-ACP added to gum, even sugarcontaining gum, was able to arrest progression and increase significantly the remineralization of subsurface enamel lesions. ${ }^{29}$

So, scientific evidence shows that the consumption of CPP-ACP gum is a low- cost, easy and effective treatment for early enamel caries.

\section{Caries infiltration with low viscosity resin}

After conventional caries-sealing, resin infiltration rises as an innovative approach to arrest progression of enamel caries lesions. ${ }^{30}$ The aim of resin infiltration is to soak up the porous lesion body with a low-viscosity resin (infiltrant) that is subsequently hardened with blue light. ${ }^{31}$ Thereby, diffusion pathways for cariogenic acids are blocked, and lesions are sealed and the progression is arrested.

In a recent 18-month clinical observation, ${ }^{32} 22$ young adults representing 29 pairs of interproximal lesions with radiological extension into the inner half of enamel or the outer third of dentin were randomly allocated to two treatment groups. Treatment was performed by a single trained investigator at the Charite-University Hospital Berlin. A rubber dam was applied to achieve dry working conditions. In the test group, allocated teeth were slightly separated by plastic wedges that had been flattened by a scalpel to leave space below the contact point. A piece of polyurethane foil was placed in the contact area with a plastic holder to protect the adjacent tooth. A $15 \% \mathrm{HCl}$ etching gel was applied by syringe in the area below the contact point for $120 \mathrm{sec}$. Subsequently, the gel was washed off with air-water-spray for $30 \mathrm{sec}$. The lesion was desiccated by air-blowing for $10 \mathrm{sec}$, application of ethanol for $10 \mathrm{sec}$, and air-blowing again for $10 \mathrm{sec}$. An infiltrant was applied with another plastic holder. After 5 min of penetration time, excess material was removed by airblowing and flossing, and the resin was light-cured for 1 
min from the buccal, occlusal, and oral aspects. The infiltration step was repeated once with a penetration time of 1 min to infiltrate remaining porosities. To avoid behavioral changes of participants with regard to oral hygiene, we did not inform them about the treatment allocation of their teeth. To ensure blinding, it was performed a placebo treatment on control teeth: instead of $\mathrm{HCl}$-gel and infiltrant, water was used. Fluoridation, oral hygiene, and dietary instructions were given to the patients. The outcome after 18 months was radiographic lesion progression assessed by digital subtraction radiography. While only $7 \%$ of the infiltrated lesions showed progression, $37 \%$ of those assigned to the control group presented caries progression, so that infiltration of interproximal caries lesions was effective in reducing lesion progression.

Although conventional caries-sealing has been found to be effective in arresting progression of enamel interproximal caries lesions, ${ }^{33}$ with this novel infiltration technique, the diffusion barrier is created inside the lesion and not on the surface. This facilitates clinical application, especially in the interproximal space, ${ }^{34}$ since no temporary tooth separation is required.

In comparison with the conventional restorative approach, both the above-mentioned treatments for either enamel smooth surface or especially interproximal enamel lesions allow a great conservation of sound tooth structure. When a restorative procedure is performed in approximal areas, substantial amounts of sound tissue have to be removed to assess caries lesions. Moreover, the first invasive intervention brings the tooth into a circle of treatment and re-treatment, often leading to crowns and implants irrespective of how well the first filling was prepared. ${ }^{35}$ In this view, the minimally invasive dentistry approach claims that nonsurgical treatments for caries lesions should be preferred to invasive ones, in order to increase the tooth's longevity in the mouth. However, if any sign of caries progression can be observed after a nonsurgical treatment for enamel lesions, the invasive one must be performed.

\section{ARRESTING CARIES LESIONS IN DENTIN THROUGH A NONSURGICAL APPROACH}

Investigations showed that carious lesions in dentin continue to be treated by most dentists us- ing an invasive approach because of the belief that remineralization or arrest of these lesions is not possible. ${ }^{36,37}$ Nevertheless, since 1998 it has been shown that composite restorations placed over frank cavitated lesions arrested their progression for 10 years. ${ }^{38}$ Recently, results obtained by Alves et al ${ }^{39}$ corroborated those from the aforementioned investigation, since even after partial carious dentin removal, progression of deep caries lesions could also be arrested. Furthermore, a deposition of tertiary dentin and a mineral gain was observed by radiological analysis. In both investigations, caries progression was controlled by restriction of nutrients for bacterial metabolism inside the lesion through cavity sealing. ${ }^{39}$ These findings corroborate the statement that, once acid production by the microbial metabolism is controlled, the caries process is controlled as well, regardless of the presence of bacteria in the dental tissue. ${ }^{38,40}$ Therefore, isolation of microorganisms from the oral environment by cavity sealing is enough to prevent lesion progression.

The option to restore a cavitated caries lesion may even be accepted, since an open cavity is found in these cases. On the other hand, dentin caries can result in visually non-cavitated lesions, which present clinically intact enamel over the bodies of the lesions. ${ }^{41}$ Some investigations have shown that invasive treatment may be recommended for the management of dentin non-cavitated carious lesions due to the lack of evidence indicating that dentin caries can be arrested. ${ }^{42,43}$ However, given the results obtained involving cavitated lesions, ${ }^{38,39}$ it can be suggested that non-cavitated caries may also be arrested using a sealant material over the visually non-cavitated enamel. When dentists perform a surgical treatment for clinically non-cavitated lesions in dentin by means of burs, a great amount of occlusal "sound" enamel needs to be removed to assess the body of the lesion.

Researchers at the Federal University of Rio Grande do Norte have investigated in vivo whether sealing the enamel over non-cavitated dentin caries lesions with fissure sealants would be able to arrest their progression. In the first time, ${ }^{44} 60$ teeth with clinically non-cavitated occlusal caries radiographically located beneath the enamel-dentin junction (radiolucent area) were selected among patients presenting a moderate to high risk of caries. The teeth were randomly divided into two 
groups of 30 teeth each: an experimental group receiving an application of a resin-based light-cure sealant/guide for oral hygiene and a control group which received only the guide for oral hygiene. After complete isolation with rubber dam, the tooth to be sealed was submitted to pumice and water prophylaxis with a Robinson brush. The occlusal surface was then etched with $37 \%$ phosphoric acid for $30 \mathrm{sec}$, washed with an air/water spray for $1 \mathrm{~min}$ and dried with an air stream according to manufacturer recommendations. Next, a resin-based light-curing sealant was applied with a dental probe to allow better flow of the material through the pits and fissures to be sealed. The sealant was then light-cured for $20 \mathrm{sec}$, and then sealant coverage was evaluated by visual inspection using a dental probe in order to detect any pit or fissure region not covered with the resin material. Occlusal contacts were examined using carbon paper to eliminate possible premature contacts. A single trained and experienced operator performed all sealings. Caries progression was monitored by clinical and radiographic examination at 4-month intervals over a period of 12 months. In addition, marginal integrity of the sealant was evaluated in the experimental group. The results showed that clinical and radiographic caries progression was significantly more frequent in control teeth when compared to the experimental group. Therefore, the resin-based light cure pit and fissure sealant was found to be effective in arresting progression of the carious lesions analyzed, suggesting that this intervention may replace the invasive treatment traditionally used for teeth with dentin noncavitated caries.

Following this theory, another clinical trial ${ }^{45}$ was conducted in an attempt to verify if a glass ionomer sealant could also arrest the progression of these dentin non-cavitated caries lesions, since glass ionomers are capable of forming chemical bonds with dental substrates in addition to releasing fluoride. ${ }^{46}$ Fifty-one teeth with clinically noncavitated occlusal caries radiographically located beneath the enamel-dentin junction Iradiolucent area) were selected among patients presenting a moderate to high risk of caries. The teeth were randomly divided into two groups: an experimental group receiving an application of a conventional glass ionomer/guide for oral hygiene and a control group not submitted to any clinical inter- vention (receiving only the guide for oral hygiene). After complete rubber dam isolation, the tooth to be sealed was submitted to pumice and water prophylaxis with a Robinson brush. Afterwards, a conventional high viscosity glass ionomer cement was used as a pit and fissure sealant. The glass ionomer cement was prepared, inserted with a dental explorer and adjusted on the occlusal surface in such a way that it remained restricted to the sulcus, thus preventing overflow and wear and tear due to occlusion and mastication. The sealant was protected with a light polymerized adhesive to prevent syneresis and imbibition. Sealant coverage was then evaluated by visual inspection using a dental explorer in order to detect any pit or fissure region not covered with the resin material. Occlusal contacts were examined using carbon paper to eliminate possible premature contacts. A single trained and experienced operator performed all sealing procedures of the study in accordance with the recommendations of the glass ionomer cement manufacturer. Caries progression was monitored by clinical and radiographic examination at 4-month intervals over a period of 12 months. In addition, marginal integrity of the glass ionomer sealant was evaluated in the experimental group. Clinical examination showed no statistical difference between the groups, so that sealed or unsealed teeth presented hypersensitivity or cavitation clinically. On the other hand, sealed teeth presented statistically significant lower caries progression when analyzed by radiographic examination. Thus, a glass ionomer sealant over non-cavitated occlusal caries lesions in dentin may not be sufficiently effective in arresting their progression. This fact might be attributed to the low mechanical strength of conventional glass ionomer cements (GICs) in oral environment, leading to macroscopic loss. Although some microscopic remnants of GICs can be present in the bottom of fissures after macroscopic loss, this is not enough to protect the fissure surface, leading it to clinical signs of progression as it was observed above. ${ }^{45}$

Therefore, it seems to be reasonable to choose a resin-based light-cure sealant to seal pits and fissures over dentin non-cavitated caries lesions. However, it must be pointed out that the loss of the sealant may occur after 12 months, ${ }^{44}$ so that clinicians should attempt to replace it if no evidence 
of caries progression is observed or to restore it if any sign of caries progression is detected.

\section{CONCLUSIONS}

In the last 100 years, dentistry has shown a great change in the approaches adopted to treat caries lesions. The effectiveness of nonsurgical methods to treat enamel lesions is fully recognized, and these methods can be used by clinicians worldwide. Concerning lesions in dentin, even visibly non-cavitated ones receive recommendations for surgical interventions due to the lack of evidence indicating that dentin caries can be arrested without surgical intervention. However, results obtained by means of recent clinical trials highlighted the possibility of treating noncavitated caries lesions in dentin by sealing the pits and fissures with a resin-based, light-cured sealant.

The paradigm as proposed by G. V. Black must be consigned as part of the history of dentistry. Although invasive treatment might be needed after some years of nonsurgical treatment for either enamel or dentin lesions to arrest the caries progression, potential benefits have already been found to result from conserving structure by delaying intervention or minimizing the operative procedure. The above-mentioned non-invasive or microinvasive treatments for caries lesion in enamel and dentin must be preferred in an attempt to preserve a higher amount of dental tissues.

\section{REFERENCES}

1. Bader JD, Shugars DA. The evidence supporting alternative management strategies for early occlusal caries and suspected occlusal dentinal caries. J Evid Base Dent Pract 2006;6:91-100.

2. Beauchamp J, Caufield PW, Crall JJ, et al. Evidence-based clinical recommendations for the use of pit-and-fissure sealants: a report of the America Dental Association council on scientific affairs. Dent Clin N Am 2009;53:131-147.

3. Paris S, Meyer-Lueckel H, Kielbassa AM. Resin infiltration of natural caries lesions. J Dent Res 2007;86:662-666.

4. Morgan MV, Adams GG, Bailey DL, et al. The anticariogenic effect of sugar-free gum containing CPP-ACP nanocomplexes on approximal caries determined using digital bitewing radiography. Caries Res 2008;42:171-184.

5. Kuisch VK. Dental caries: a new look at an old disease. Inside Dent 2009;5:60-65.
6. Feacherstone JD. Dental caries: a dynamic disease process. Aust Dent J 2008;53:286-291.

7. Diefenderfer KE, Stahl J. Caries remineralization therapy: implications for dental readiness. Mil Med 2008;173:48-50.

8. Maltz M, Jardim JJ, Alves LR. Health promotion and dental caries. Braz Oral Res 2010;24(Spec Issue 1):18-25.

9. Sharma S, Kugel G. Amorphous calcium phosphate sealants - the potential to remineralize. Inside Dent 2009;5:7880.

10. Bartlett DW, Bureau GP, Anggiansah A. Evaluation of the $\mathrm{pH}$ of a new carbonated soft drink beverage: an in vivo investigation. J Prosthodont 2003;12:21-25.

11. Khan AA, Jain SK, Shrivastav A. Prevalence of dental caries among the population of Gwalior (India) in relation of different associated factors. Eur J Dent 2008;2:81-85.

12. Kugel G, Arsenault P, Papas A. Treatment modalities for caries management, including a new resin infiltration system. Compend Contin Educ Dent 2009;30(Spec Issue 3):1-10.

13. Kidd EA, Fejerskov 0. What Constitutes Dental Caries? Histopathology of carious enamel and dentin related to the action of cariogenic biofilms. J Dent Res 2004;83/Spec Issue C):C35-C38.

14. Mount GJ. A new paradigm for operative dentistry. Aust Dent J 2007;52:264-270.

15. Black GV. Operative Dentistry. Chicago: Medico-Dental Publishing, 1908:121.

16. Massler M. Changing concepts in the treatment of carious lesions. Br Dent J 1967;123:547-548.

17. ten Cate JM, Duijsters PP. Alternating demineralization and remineralization of artificial enamel lesions. Caries Res $1982 ; 16: 201-210$

18. Featherstone JB. The continuum of dental caries - evidence for a dynamic disease process. J Dent Res 2004;83/Spec Issue C):39-42.

19. Featherstone JB, ed. Clinical aspects of the de/remineralization of teeth. Adv Dent Res 1995;9:1-340.

20. Brännström M. Dentin and pulp in restorative dentistry. London: Wolfe Medical Publications Ltd., 1982.

21. Reynolds EC, Walsh IJ. Additional aids to the remineralization of tooth structure. In: Mount GJ, Hume WR, eds. Preservation and restoration of tooth structure. $2^{\text {nd }}$ edn. Brisbane: Knowledge Books and Software, 2005:111-118.

22. Ngo HC, Mount GJ, Mclntyre J, et al. Chemical exchange between glass-ionomer restorations and residual carious dentine in permanent molar: an in vivo study. $J$ Dent 2006;34:608-613.

23. Pitts NB. Modern concepts of caries measurement. J Dent Res 2004;83(Spec Issue C):43-47. 
24. McComb D. Systematic review of conservative operative caries management strategies. J Dent Educ 2001;65:11541161.

25. Zero DT. Dentifrices, mouthwashes, and remineralization/ caries arrestment strategies. BMC Oral Health 2006;6:S9.

26. Mjör IA, Holst D, Eriksen HM. Caries and restoration prevention. J Am Dent Assoc 2008;139:565-570

27. Reynolds EC, Cain CJ, Shen P, et al. Retention in plaque and remineralization of enamel lesions by various forms of calcium in a mouthrinse or sugar-free chewing gum. J Dent Res 2003;82:206-211.

28. Manton DJ, Walker GD, Cai F, Cochrane NJ, Shen P, Reynolds EC. Remineralization of enamel subsurface lesions in situ by the use of three commercially available sugar-free gums. Int J Paediatr Dent 2008;18:284-290.

29. Walker GD, Cai F, Shen P, et al. Casein phosphopeptideamorphous calcium phosphate incorporated into sugar confections inhibit the progression of enamel subsurface lesions in situ. Caries Res 2010;44:33-40.

30. Paris S, Meyer-Lueckel H. Inhibition of caries progression by resin infiltration in situ. Caries Res 2010;44:47-54.

31. Paris S, Meyer-Lueckel $H$, Colfen $H$, et al. Resin infiltration of artificial enamel caries lesions with experimental light curing resins. Dent Mater J 2007;26:582-588.

32. Paris S, Hopfenmuller W, Meyer-Lueckel H. Resin infiltration of caries lesion: an efficacy randomized trial. $J$ Dent Res 2010; doi: 10.1177/0022034510369289

33. Martignon S, Ekstrand KR, Ellwood R. Efficacy of sealing proximal early active lesions: an 18-month clinical study evaluated by conventional and subtraction radiography. Caries Res 2006;40:382-388.

34. Phark JH, Duarte S Jr, Meyer-Lueckel H, et al. Caries infiltration with resins: a novel treatment option for interproximal caries. Compend Contin Educ Dent 2009;30/Spec Issue 3):13-17.

35. ten Cate JM. Remineralization of deep enamel dentin caries lesions. Aust Dent J 2008;53:281-285.

36. Doméjean-Orliaguet S, Tubert-Jeannin S, Riordan PJ, et al. French dentists' restorative treatment decisions. Oral Health Prev Dent 2004;2:125-131.

37. Traebert J, Wesoloski Cl, Lacerda JT, et al. Threshold of restorative decision in dental caries treatment among dentists from small Brazilian cities. Oral Health Prev Dent 2007;5:131-135.

38. Mertz-Fairhurst EJ, Curtis-Jr. JW, Ergle JW, et al. Ultraconservative and cariostatic sealed restorations: results at year 10. J Am Dent Assoc 1998;129:55-66.
39. Alves LS, Fontanella V, Damo AC, et al. Qualitative and quantitative radiographic assessment of sealed carious dentin: a 10-year prospective study. Oral Surg Oral Med Oral Pathol Oral Radiol Endod 2010;109:135-41.

40. Maltz M, Oliveira EF, Fontanella V, et al. A clinical, microbiologic, and radiographic study of deep lesions, after incomplete caries removal. Quintessence Int 2002;33:151-159.

41. Weerheijm KL, van Amerongen WE, Eggink CO. The clinical diagnosis of occlusal caries: a problem. ASDC J Dent Child 1989;56:196-200.

42. Murdoch-Kinck C, McLean M. Minimally invasive dentistry. $J$ Am Dent Assoc 2003;134:87-95.

43. Brostck A. Early diagnosis and minimally invasive treatment of occlusal caries - a clinical approach. Oral Health Prev Dent 2004;2(Suppl 1):313-319.

44. Borges BC, Campos GB, da Silveira AD, de Lima KC, Pinheiro IV. Efficacy of a pit and fissure sealant in arresting dentin non-cavitated caries: a 1-year follow-up, randomized, single-blind, controlled clinical trial. Am J Dent 2010;23:311-316.

45. Silveira AD, Borges BC, Varela HA, et al. Progression of non-cavitated lesions in dentin through a nonsurgical approach: a preliminary 12-month clinical observation. Eur $J$ Dent 2010, in press.

46. Uysal T, Amasyali M, Koyuturk AE, et al. Efficiency of amorphous calcium phosphate-containing orthodontic composite and resin modified glass ionomer cement on demineralization evaluated by a new laser fluorescence device. Eur J Dent 2009;3:127-34. 